\title{
How can communities and organisations improve their health literacy?
}

\author{
Jane Lloyda, Louise Thomas ${ }^{a, c}$, Gawaine Powell-Davies ${ }^{a}$, Regina \\ Osten ${ }^{a, b}$ and Mark Harris ${ }^{a}$
}

a Centre for Primary Health Care and Equity, UNSW Sydney, Australia

${ }^{b}$ Agency for Clinical Innovation, Sydney, NSW, Australia

c Corresponding author: Louise.Thomas@unsw.edu.au

\section{Article history}

Publication date: June 2018

Citation: Lloyd J, Thomas L, PowellDavies G, Osten R, Harris M. How can communities and organisations improve their health literacy? Public Health Res Pract. 2018;28(2):e2821809. https://doi. org/10.17061/phrp2821809

\section{Key points}

- Health literacy is a dynamic, twoway relationship, affected by both organisational and community factors

- The 'teach-back' method is a useful way of checking patient understanding, but it is not a communication strategy in itself

- Interventions are needed to develop organisational practice and community capacity. Examples of these interventions have been developed in Australia in some Local Health Districts and Primary Health Networks

\section{Abstract}

Definitions of health literacy have tended to focus on the abilities of patients and communities, rather than on the ability of the health system and its services to respond to patients' different levels of health literacy. However, health literacy is increasingly being recognised as part of a dynamic, two-way relationship, affected by both organisational factors (e.g. tailoring of communication and care to patients' needs) and community factors (e.g. individuals' ability to perceive and seek care). Developing a more comprehensive understanding of health literacy is an important step towards improving health literacy.

Most health literacy interventions described in the literature tend to be small and focused on either organisational or community aspects of health literacy rather than addressing both sides. However, some good examples can be found in Local Health Districts and Primary Health Networks in New South Wales (NSW), Australia, of health literacy interventions that are multidimensional and address both organisational and community health literacy.

Although progress is being made, gaps in knowledge remain. A deeper understanding of the intersection between health literacy, culture and language is needed, as well as identification of effective communication strategies after patient comprehension has been assessed using strategies such as 'teach-back'. The teach-back method can be used to check patient understanding, but it is not a communication strategy in itself. If teachback shows that the patient has not understood, clinicians can employ communication strategies such as limiting discussion to two or three points, or using visual aids. If these are not effective, extended family networks and the use of patient navigators may be required. These health literacy interventions address both organisational and community aspects. More work is needed to evaluate such interventions, in particular their impact on health literacy and appropriate and timely access to healthcare. 


\section{Introduction}

\section{What is health literacy and why do we care} about it?

Health literacy is the set of knowledge, attitudes, skills and networks that individuals, families and communities need for managing their health. ${ }^{1}$ Adequate health literacy supports people to maintain good health, manage minor illnesses, access and make wise use of health services, follow agreed treatments and self-care routines, and advocate for a healthy society. ${ }^{2}$ Achieving these goals requires an understanding of health and the factors that affect it, as well as an understanding of health services and how they work, and the motivation to play an active role in managing one's health. All health-related tasks, from maintaining good health through to managing ill health and receiving healthcare, require some degree of health literacy. ${ }^{3}$

\section{Shift from sole patient focus to recognising the role that communities and organisations can play}

Concepts about the meaning of health literacy are contested. ${ }^{1}$ Definitions have tended to focus on the health literacy of patients and communities, rather than the ability of the health system and its services to respond to the different health literacy needs of patients. However, organisational health literacy is important in its own right and can be defined as both the organisation's efforts to support patients and community health literacy, and the organisation's own cultural and community literacy. ${ }^{4}$

There is increasing international recognition of the importance of reducing the complexity of health systems, and improving the way services and information are provided. The US Institute of Medicine (now the National Academy of Medicine) was the first to advocate for this shift in thinking away from a patient-centric view of health literacy. ${ }^{5}$ In addition to this responsibility to provide services that meet the needs of people with low health literacy, the health system also has a responsibility to build up patient health literacy ${ }^{6}$ by, for example, tailoring communication and regularly checking for understanding. As such, health literacy is part of a dynamic, two-way relationship. Like the Levesque 'access' model', which views healthcare access as being influenced by both provider and patient characteristics, health literacy can also be understood as being affected by organisational and community factors.

Community-based health literacy initiatives focus on empowering individuals to gain health knowledge and to act on it within their social and cultural context. ${ }^{8}$ Synthesising these social-contextual and personal resources is thought to result in health empowerment. ${ }^{9}$ Personal resources such as health knowledge, and the capacity to solve problems and make meaningful changes can be developed by providing health education in a culturally and linguistically appropriate manner. Social and contextual resources such as contacts and support networks can be developed in a group setting with an educator who is skilled in building trust and connection. ${ }^{9}$ Community-based approaches have the potential to facilitate the exchange and co-creation of health knowledge, by community members, within their own context. ${ }^{8}$

\section{Interventions}

A nuanced and comprehensive understanding of health literacy is an important step towards improving health literacy, but is insufficient for generating change.

Developing community capacity and organisational practice requires interventions that address both the community and organisational aspects affecting health literacy. This perspective article identifies how community and organisational health literacy interventions can be applied in practice by describing examples within Local Health Districts (LHDs) and Primary Health Networks (PHNs) in New South Wales (NSW), Australia.

\section{Health literacy interventions and how they might be used in LHDs and PHNs}

The majority of health literacy research has tended to focus on defining health literacy rather than developing and evaluating interventions. Of the health literacy interventions that have been described in the literature, most have tended to focus on increasing the knowledge, skills and abilities of specific target populations and have tended to be small and one-dimensional, rather than multidimensional.

In 2012, the Australian Commission on Safety and Quality in Health Care (ACSQHC) conducted a stocktake of health literacy interventions in Australia. ${ }^{10}$ The most common interventions (47\% of 229) were those that improved the communication of health information. These interventions largely involved health service staff or subject experts developing information resources designed for a particular target audience. Interventions that aimed to modify the health service environment and to develop policies or frameworks for improving health literacy were less common. However, these multidimensional interventions are important for addressing both community and organisational factors.

The stocktake did not comment on the effectiveness of these interventions, but a systematic review by Berkman et al. ${ }^{11}$ described the following effective characteristics of health literacy interventions: high intensity, based on theory, pilot tested before implementation, delivered by a health professional and with an emphasis on building skills. 
The Illawarra Shoalhaven Local Health District (ISLHD) Health Literacy Framework ${ }^{12}$ is a good example of a health literacy intervention that addresses both organisational and community health literacy. Strategies within the framework include: plain language consumer information, systems and resources to drive and support change, health literacy champions, effective communication (teach-back), access and wayfinding, and a patient information portal. Considering, for example, the strategies of plain language consumer information and a patient information portal, the organisation must ultimately make these resources available to communities. However, increasingly, organisations are developing these resources in a way that relies on community input and co-design. Engaging communities in this process can build their health literacy and improve the appropriateness and accessibility of the consumer information and patient information portal. Once the patient information is available, it is more likely to enable patients with low health literacy to access the information they need in the format they can understand, which in turn improves their health literacy.

Examples of interventions developed in $\mathrm{PHNs}$ include the Health Literacy Guide developed by the Hunter New England and Central Coast $\mathrm{PHN}^{13}$ and the joint health literacy project between the North Coast Primary Health Network and the Northern NSW LHD. ${ }^{14}$ The Northern NSW Health Literacy Project aims to improve wayfinding for patients, and support providers and services to tailor their communication to patients' health literacy. A website provides information and resources about health literacy, and health literacy workshops are available for consumers and health professionals.

Evaluating the impact of interventions such as these on health literacy and access to healthcare is an important next step. Evaluations of organisational health literacy interventions will differ depending on the type of intervention, but could consider the impact of the intervention on policy and practice, changes to provider knowledge, attitudes and skills, and the effect on patient experience. The recently established Western Sydney LHD Health Literacy Hub is aiming to take this next step, by seeking to develop and test interventions and practical solutions to improve health literacy in a range of community and healthcare settings. ${ }^{15}$

\section{Supportive structures and incentives}

Health literacy interventions require supportive structures to be in place within the community and organisations. Community interventions can be supported through strong structure and leadership, and by an active network of people to facilitate change. It may be more difficult to incentivise these community-level interventions compared with organisational interventions because empowering individuals within their social and cultural context is a more amorphous and less-regulated setting. There is rarely one community, rather many communities within specific locations and demographics. Organisationallevel interventions can be supported by having health literacy champions and recognising health literacy within the organisational framework. Further, having standards and accreditation processes can incentivise the active facilitation of health literacy at an organisational level. This is the case in LHDs and PHNs, where health literacy interventions can be supported and reinforced by the National Safety and Quality Health Service Standards. ${ }^{16}$ The standards have an increasing focus on health literacy and, more broadly, an emphasis on supporting consumers' understanding of and engagement with their healthcare. Organisations such as PHNs have the potential to play an important role in improving community knowledge and the cultural competence of services.

\section{Gaps in knowledge}

Although there is increasing acceptance of the need for organisational and community health literacy interventions, there are still gaps in knowledge.

It is generally understood that organisational health literacy can be improved by keeping health routines and communication simple, and regularly assessing comprehension of clinical content with patients, for example through 'teach-back' techniques. The 'teachback' method is used to confirm patient understanding Patients are asked to communicate what they have understood from the clinician as a test of how well the clinician communicated and to identify if further explanation is required. However, techniques such as these do not identify other ways to communicate that may be more effective.

Further, to improve organisational and community health literacy, the intersection between health literacy, culture and language requires additional investigation. Language can be an obvious barrier to health literacy, especially if a person is not literate in their primary language. A skilled healthcare interpreter can help overcome a language barrier, but just as important and less obvious, and harder to address, may be differences in culture and understanding. However, the evidence for effective interventions that take account of health literacy and cultural competence is limited.

Interventions described within the ACSQHC stocktake of health literacy interventions in Australia ${ }^{10}$ that were targeted towards people from culturally and linguistically diverse backgrounds were focused on translating, developing or adapting material for different language groups. Although these interventions might help to overcome language barriers, they may not result in improved understanding or access to care unless health literacy and cultural competency are also taken into consideration. Providing translated written material alone will not be sufficient for a patient who is literate in the translated language but has poor health literacy. 


\section{Conclusion}

There is increasing recognition that community-based initiatives to improve critical health literacy are needed to enable priority population groups to make informed health decisions and take action to improve and/or maintain their health. At the same time, organisational and servicebased initiatives are needed to ensure responsiveness to the various health literacies and cultural and language needs of patients. Interventions to address community and organisational factors are emerging, but more are needed, and their impact on health literacy and timely access to health services needs to be evaluated.

\section{Acknowledgements}

The authors acknowledge the contribution of Terry Findlay, Centre for Primary Health Care and Equity, UNSW Sydney, to the design of the review. The research on which this manuscript is based was funded by the Agency for Clinical Innovation.

\section{Peer review and provenance}

Externally peer reviewed, not commissioned

\section{Competing interests}

$\mathrm{MH}$ is a board member of the Central and Eastern Sydney PHN.

\section{Author contributions}

$\mathrm{JL}$ led the design of the review and led a component of the analysis, and contributed to drafting and editing the manuscript. LT contributed to the analysis and was responsible for drafting and editing the manuscript. GP-D led components of the analysis and contributed to editing the manuscript. RO contributed to the design of the review, commented on the analysis and helped edit the manuscript. $\mathrm{MH}$ contributed to the design of the review and led a component of the analysis.

\section{References}

1. Sorensen K, Van den Broucke S, Fullam J, Doyle G, Pelikan J, Slonska Z, et al. Health literacy and public health: a systematic review and integration of definitions and models. BMC Public Health. 2012;12(80).

2. von Wagner C, Steptoe A, Wolf M, Wardle J. Health literacy and health actions: a review and a framework from health psychology. Health Educ Behav. 2009;36(5):860-77.

3. Paasche-Orlow MK, Wolf MS. The causal pathways linking health literacy to health outcomes. Am J Health Behav. 2007;31 Suppl 1:S19-26.

4. Brach C, Keller D, Hernandez LM, Baur C, Parker R, Dreyer B, et al. Ten attributes of health literate health care organizations. Washington DC: Institute of Medicine; 2012 [cited 2018 Apr 18]. Available from: https://nam. edu/wp-content/uploads/2015/06/BPH_Ten_HLit_ Attributes.pdf

5. Institute of Medicine Committee on Health Literacy. Health literacy: a prescription to end confusion. NielsenBohlman L, Panzer AM, Kindig DA, editors. Washington DC: The National Academies Press; 2004. 366 p.

6. Nutbeam D. The evolving concept of health literacy. Social SciMed. 2008;67(12):2072-8.

7. Levesque JF, Harris MF, Russell G. Patient-centred access to health care: conceptualising access at the interface of health systems and populations. Int J Equity Health. 2013;12(1):18.

8. de Wit L, Fenenga C, Giammarchi C, di Furia L, Hutter I, de Winter A, Meijering L. Community-based initiatives improving critical health literacy: a systematic review and meta-synthesis of qualitative evidence. BMC Public Health. 2017;18(1):40

9. Crawford Shearer NB. Health empowerment theory as a guide for practice. Geriatr Nurs. 2009;30(2 Suppl):4-10.

10. Australian Commission on Safety and Quality in Health Care. Health literacy stocktake: consultation report. Sydney: ACSQHC; 2012 [cited 2018 Apr 18]. Available from: www.safetyandquality.gov.au/wp-content/ uploads/2012/01/Health-Literacy-Stocktake-ConsultationReport1.pdf

11. Berkman ND, Sheridan SL, Donahue KE, Halpern DJ, Crotty K. Low health literacy and health outcomes: an updated systematic review. Ann Intern Med. 2011;155(2):97-107.

12. Illawarra Shoalhaven Local Health District. Health Literacy Framework 2012-2015: a plan for becoming a health literate organisation. Warrawong: ISLHD; 2012 (revised 2014) [cited 2018 Apr 18]. Available from: www.cec. health.nsw.gov.au/_data/assets/pdf_file/0010/259066/ islhdhealthliteracyframework.pdf 
13. Hunter New England and Central Coast Primary Health Network. HNECC health literacy guide. Newcastle: HNECC PHN [cited 2018 Apr 18]. Available from: hneccphn.com.au/media/14219/014836-hnecc-healthliteracy-guide-10.pdf

14. Northern NSW Local Health District. About the Northern NSW Health Literacy Project. Lismore: NNSWLHD; 2017 [cited 2018 Apr 18]; [about 2 screens]. Available from: healthliteracy.nnswlhd.health.nsw.gov.au/about/
15. Integrated health: moving to the future. Forum report. 2017 [cited 2018 Apr 18]. Available from: www. betterhealthtogether.com.au/themes/default/basemedia/ content/files/IH-Forum-Report-FINAL-SEPT-2017.PDF

16. Australian Commission on Safety and Quality in Health Care. National safety and quality health service standards, second edition. Sydney: ACSQHC; 2017 [cited 2018 May 15]. Available from: www. safetyandquality.gov.au/our-work/assessment-to-thensqhs-standards/nsqhs-standards-second-edition/

\section{Copyright: (c) (i) (2)}

(C) 2018 Lloyd et al. This article is licensed under the Creative Commons Attribution-NonCommercial-ShareAlike 4.0 International Licence, which allows others to redistribute, adapt and share this work non-commercially provided they attribute the work and any adapted version of it is distributed under the same Creative Commons licence terms. See: www.creativecommons.org/licenses/by-nc-sa/4.0/ 